\title{
Práticas sexuais e cuidados relacionados à saúde sexual de graduandos de enfermagem frente às infecções sexualmente transmissíveis
}

\author{
Sexual practices and care related to sexual health of nursing undergraduates against sexually \\ transmitted infections \\ Prácticas sexuales y cuidados relacionados a la salud sexual de estudiantes de enfermería frente a \\ las infecciones sexualmente transmisibles

\section{Thelma Spindola', Carolina Passos SodréII, Claudia Silvia Rocha OliveiraIII Barbara Ingênito de Oliveira $^{\mathrm{IV}}$, Rosana Santos Costa Santanav Nathalia Lourdes Nepomuceno de Oliveira Andrévi}

\begin{abstract}
Resumo: Objetivo: identificar as práticas sexuais e os cuidados com a saúde sexual de graduandos de enfermagem frente às infecções sexualmente transmissíveis. Método: estudo transversal descritivo quantitativo, realizado em duas instituições de ensino superior do Rio de Janeiro, com 250 estudantes. Para a análise das variáveis empregou-se a estatística descritiva. Resultados: a maioria, $132(52,8 \%)$ tem idades entre 18 - 21 anos; sendo $217(86,8 \%)$ do sexo feminino. Entre os participantes 185 (74.0\%) possuem vida sexual ativa. Desses, 113 $(61,1 \%)$ utilizaram o preservativo na sexarca e $107(57,8 \%)$ informaram fazer uso em todos os intercursos sexuais. Em relação ao cuidado com a saúde, $110(44,0 \%)$ já fizeram teste para detectar o HIV. Entre as mulheres, 125(57,6\%) fizeram o exame Papanicolau. Conclusão: pode-se inferir que os graduandos de enfermagem, são vulneráveis às infecções sexualmente transmissíveis e adotam comportamentos de risco em suas práticas sexuais.

Descritores: Vulnerabilidade em saúde; Adulto jovem; Doenças sexualmente transmissíveis; Enfermagem
\end{abstract}

\footnotetext{
I Enfermeira. Doutora em Enfermagem. Professora Associada do Departamento de Fundamentos de Enfermagem e do Programa de Pósgraduação em Enfermagem. Universidade do Estado do Rio de Janeiro. Rio de Janeiro. Brasil. E-mail: tspindola.uerj@gmail.com. Orcid https://orcid.org/0000-0002-1785-5828.
}

II Enfermeira. Graduada em Enfermagem pela Universidade do Estado do Rio de Janeiro. Universidade do Estado do Rio de Janeiro, Rio de Janeiro, Brasil. E-mail: c.sodrepassos@gmail.com. Orcid - https://orcid.org/0000-0002-9819-2156.

III Enfermeira. Mestranda em Enfermagem pela Universidade do Estado do Rio de Janeiro. Universidade do Estado do Rio de Janeiro. Rio de Janeiro, Brasil. E-mail: enf.claudiaoliveira@gmail.com. Orcid - https://orcid.org/0000-0002-0794-7366.

IV Enfermeira. Mestre em Enfermagem pela Universidade do Estado do Rio de Janeiro. Universidade do Estado do Rio de Janeiro. Rio de Janeiro, Brasil. E-mail: barbaraingenito@hotmail.com. Orcid - https://orcid.org/0000-0003-4765-6667.

V Enfermeira. Mestre em Enfermagem pela Universidade do Estado do Rio de Janeiro. Universidade do Estado do Rio de Janeiro. Rio de Janeiro, Brasil. E-mail: zana.s.costa@gmail.com. Orcid - https://orcid.org/0000-0002-4342-6322.

VI Enfermeira. Graduada em Enfermagem pela Universidade do Estado do Rio de Janeiro. Universidade do Estado do Rio de Janeiro, Rio de Janeiro, Brasil. E-mail: nathnepofenf@gmail.com. Orcid - https://orcid.org/0000-0001-8188-6701 
Práticas sexuais e cuidados relacionados à saúde sexual de graduandos de enfermagem.... $\mid 2$

\begin{abstract}
Aim: to identify the sexual practices and the sexual health care of nursing undergraduates against sexually transmitted infections. Method: a quantitative descriptive cross - sectional study, carried out in two higher education institutions of Rio de Janeiro, with 250 students. Descriptive statistics were used to analyze the variables. Results: a majority of $132(52.8 \%)$ were between 18 and 21 years of age; of which 217 (86.8\%) were female. Among the participants, 185 (74.0\%) had active sex life, 113 (61.1\%) used the condom in the sexarca and 107 (57.8\%) reported using it in all sexual intercourse. Regarding health care, 110 (44.0\%) have already been tested for HIV. Among women, 125 (57.6\%) had a Pap smear. Conclusion: it can be inferred that nursing undergraduates are vulnerable to sexually transmitted infections and adopt risky behaviors in their sexual practices.
\end{abstract}

Descriptors: Vulnerability in health; Young adult; Sexually transmitted diseases; Nursing.

Resumen: Objetivo: identificar las prácticas sexuales y cuidados con la salud sexual de estudiantes de enfermería frente a las infecciones sexualmente transmisibles. Método: estudio transversal, descriptivo, cuantitativo, realizado en dos instituciones de enseñanza superior de Río de Janeiro, con 250 estudiantes. Para el análisis de las variables se empleó la estadística descriptiva. Resultados: la mayoría de los estudiantes 132 (52,8\%) tienen edades entre 18 21 años; 217 (86,8\%) son del sexo femenino. Entre los participantes, 185 (74.0\%) poseen vida sexual activa, de esos $113(61,1 \%)$ utilizaron preservativo en la sexarca y $107(57,8 \%)$ informaron hacer uso en todas las relaciones sexuales. Con relación al cuidado con la salud, $110(44,0 \%)$ ya hicieron prueba para detectar el VIH. Entre las mujeres, 125 $(57,6 \%)$ hicieron el examen Papanicolau. Conclusión: se puede inferir que los estudiantes de enfermería son vulnerables a las infecciones sexualmente transmisibles y adoptan comportamientos de riesgo en sus prácticas sexuales.

Descriptores: Vulnerabilidad en salud; Adulto joven; Enfermedades sexualmente transmisibles; Enfermería.

\title{
Introdução
}

As Infecções Sexualmente Transmissíveis (IST) são transmitidas principalmente por meio do contato sexual e sanguíneo e causadas por diferentes agentes etiológicos como vírus, bactérias e protozoários. As IST são consideradas um problema para a saúde pública, porque repercutem na saúde reprodutiva da população e podem causar infertilidade, perda fetal, morte prematura, infecções em recém-nascidos, câncer de pênis e cervical, além de ser um fator de risco para a infecção pelo vírus da imunodeficiência humana (HIV). ${ }^{1-2}$

O início da vida sexual, com frequência, ocorre na juventude e a média de idade da primeira relação é de 14,9 anos, ou seja, na adolescência. Na ocasião, a cognição e a tomada de decisão ainda estão em desenvolvimento, o que favorece para que os jovens fiquem mais vulneráveis e assumam comportamentos de risco como a prática do sexo desprotegido. ${ }^{3}$ 
Para o Estatuto da Juventude, indivíduos com idades entre 15 e 29 anos são reconhecidos como jovens. ${ }^{4} \mathrm{~A}$ juventude perpassa a puberdade, a adolescência e o início da vida adulta, fases importantes na vida, sendo um momento de transição, formação de identidade e mudanças externas advindas da puberdade. Não se restringe ao âmbito biológico, mas compreende, também, aspectos sociais e psicológicos. ${ }^{5}$

A elevada incidência na população brasileira, de pessoas soropositivas para o HIV e outras IST se apresenta como um grave problema de saúde pública. Pesquisas recentes do Ministério da Saúde apontam que no período de 2010 a 2016 foram notificados 342.531 casos de sífilis adquirida. Em 2016, a maior parte das notificações ocorreu em indivíduos de 20 a 29 anos, e entre os jovens de 13 a 19 anos, esse grupo vêm apresentando tendência de aumento desde 2010. ${ }^{6}$ Investigação ${ }^{7}$ realizada pelo Ministério da Saúde, em conjunto com o Hospital Moinhos de Vento na cidade de Porto Alegre - Rio Grande do Sul, com 7.586 participantes com idades entre 16 e 25 anos, estimou a prevalência do HPV em 54,6\% jovens. Entre os participantes, 38,4\% apresentaram HPV de alto risco para o desenvolvimento de câncer.

Estudos ${ }^{2,4}$ apontam que os jovens assumem comportamentos considerados de risco ou possuem desconhecimento dos métodos de prevenção das doenças, o que os torna vulneráveis a essas infecções. Assim, o início precoce da atividade sexual, o uso descontínuo ou incorreto de preservativos, a utilização de álcool e outras drogas, a síndrome da adolescência normal e os comportamentos influenciados pelo ambiente social do jovem, são indícios que a vulnerabilidade está associada a fatores individuais, sociais, econômicos e culturais. ${ }^{8-9}$

O termo vulnerabilidade surgiu na década de 90 associado à epidemia do HIV e representa um conjunto de condições que tornam os indivíduos suscetíveis às doenças. ${ }^{10} \mathrm{~A}$ vulnerabilidade está relacionada à concepção de bem-estar social adotado pelo Estado. ${ }^{11} \mathrm{O}$ conceito de vulnerabilidade pode ser entendido em três perspectivas: a individual, a social e a programática. A vulnerabilidade individual considera o nível de informações que cada indivíduo possui e como essas informações são assimiladas em sua vida. A vulnerabilidade social é o conjunto de fatores disponíveis na sociedade, o 
acesso às informações, serviços, cultura, dentre outros, que podem ser utilizados pelo cidadão. Já a vulnerabilidade programática é referente às ações políticas e aos serviços de saúde realizados para reduzir e controlar as enfermidades. ${ }^{12}$

Os aspectos socioeconômicos, culturais e comportamentais estão diretamente relacionados à vulnerabilidade de um grupo às IST. Assim, investigações sobre o assunto contribuem para que se obtenha maior compreensão do fenômeno e a determinação de ações estratégicas para reduzir esses agravos. Ações de prevenção das IST compreendem a redução do número de parcerias sexuais, o uso continuado dos preservativos, a busca de atendimento de saúde tão logo se observe sinais e sintomas, e a realização de exames periódicos, dentre outros. ${ }^{1}$

Nesta perspectiva, essa investigação tem o objetivo de identificar as práticas sexuais e os cuidados com a saúde sexual dos graduandos de enfermagem frente às infecções sexualmente transmissíveis.

\section{Método}

Trata-se de um estudo descritivo, transversal, de abordagem quantitativa, realizado em duas instituições de ensino superior, localizadas no município do Rio de Janeiro. Participaram 250 estudantes universitários, sendo 135 de uma universidade pública e 115 de uma instituição privada, no período de 2013/2014.

A amostra foi do tipo intencional não probabilística composta apenas por estudantes do primeiro e segundo períodos acadêmicos do curso de enfermagem. A escolha dos períodos iniciais se justifica porque ao adentrar no curso superior, os jovens são inseridos em disciplinas do ciclo básico, como fisiologia, bioquímica, anatomia e outras, e dispõem de pouca informação sobre os modos de prevenção de doenças. Houve interesse, então, em captar as informações antes que os jovens tivessem acesso a esses conteúdos. 
Como critérios de inclusão, adotaram-se estudantes de graduação em enfermagem, de ambos os sexos, com idade entre 18 e 29, regularmente matriculados no curso de enfermagem das instituições, pertencentes ao primeiro e segundo períodos acadêmicos. Foram excluídos os estudantes que não estavam presentes no período da coleta de dados.

Após a identificação dos participantes que atendiam aos critérios de inclusão, foi aplicado um questionário estruturado com 50 questões fechadas contendo variáveis socioeconômicas, comportamentais e aquelas referentes ao conhecimento e prevenção das IST. A coleta aconteceu durante os intervalos das aulas com o propósito de não atrapalhar a dinâmica na sala de aula, tendo os participantes assinado previamente o Termo de Consentimento Livre e Esclarecido (TCLE) como prevê a Resolução 466/2012.

O instrumento de coleta de dados (ICD) foi testado previamente, realizando-se um teste piloto com 10 estudantes universitários para verificar a objetividade, clareza e pertinência aos objetivos propostos. Com isso, foram feitos ajustes no questionário e os instrumentos utilizados no teste, descartados. Vale ressaltar o ICD foi adaptado e elaborado a partir de uma investigação, do Ministério da Saúde, intitulada "Pesquisa de Comportamentos, Atitudes e Práticas da População Brasileira”.

Os dados foram lançados no programa Excel da Microsoft office 2013 for Windows. As variáveis selecionadas, em sua maioria, eram qualitativas e dicotômicas. Para a análise, empregaram-se os recursos da estatística descritiva com análise univariada e os resultados foram apresentados em frequências absoluta e percentual, sendo discutidos à luz dos estudiosos da temática.

A pesquisa foi previamente submetida e aprovada pelos Comitês de Ética em Pesquisa das instituições sede da pesquisa com os pareceres número 063/2012 e 327.872/2013.

\section{Resultados}


Práticas sexuais e cuidados relacionados à saúde sexual de graduandos de enfermagem....... 6

Fizeram parte do conjunto amostral desta pesquisa 250 estudantes universitários do curso de enfermagem. Entre os participantes, mais da metade tinham idades entre 18 - 21 anos 132 (52,8\%). Quanto ao sexo, $217(86,8 \%)$ eram do sexo feminino e $33(13,2 \%)$ do masculino. Em relação à situação conjugal, $130(52,0 \%)$ não possuíam relacionamentos afetivos; $67(26,8 \%)$ tinham relacionamento estável; $16(6,4 \%)$ viviam união não estável e $37(14,8 \%)$ não responderam a questão. No aspecto religioso, $214(89,2 \%)$ praticavam uma religião; desses 96 (44,8\%) eram católicos.

No grupo investigado, $178(71,2 \%)$ residiam com os pais; 30 (12.0\%) com familiares; 18 (7,2\%) com companheiro(a); $13(5,2 \%)$ moravam sozinhos e $11(4,4 \%)$ com amigos. No tocante a situação empregatícia, $171(68,4 \%)$ não trabalhava; desse total $120(48.0 \%)$ pertencem à universidade pública e $51(20,4 \%)$ a privada.

Entre os participantes, $185(74,0 \%)$ tinham vida sexual ativa, e 133 (71,9\%) tiveram sua primeira relação sexual entre 12 e 18 anos. A Tabela 1 apresenta as práticas sexuais dos jovens universitários.

Tabela 1: Práticas sexuais dos graduandos de enfermagem e uso de preservativo. Rio de Janeiro, 2015. $\mathrm{n}=185$

\begin{tabular}{|c|c|c|c|c|c|c|c|c|}
\hline \multirow[t]{2}{*}{ Práticas Sexuais } & \multicolumn{2}{|c|}{ Sim } & \multicolumn{2}{|c|}{ Não } & \multicolumn{2}{|c|}{${ }^{*} \mathbf{N i}$} & \multicolumn{2}{|c|}{ Total } \\
\hline & $\mathrm{f}$ & $\%$ & $\mathrm{f}$ & $\%$ & $\mathrm{f}$ & $\%$ & $\mathrm{f}$ & $\%$ \\
\hline $\begin{array}{l}\text { Usaram preservativos na primeira } \\
\text { relação sexual }\end{array}$ & 113 & 61,1 & 70 & 38,0 & 2 & 1,1 & 185 & 100 \\
\hline $\begin{array}{l}\text { Usaram preservativos em todos os } \\
\text { intercursos sexuais }\end{array}$ & 107 & 58,0 & 78 & 42,2 & - & - & 185 & 100 \\
\hline $\begin{array}{l}\text { Tiveram relações sexuais com } \\
\text { parceiros fixos no último ano }\end{array}$ & 158 & 85,4 & 24 & 13,0 & 3 & 1,6 & 185 & 100 \\
\hline $\begin{array}{l}\text { Usaram preservativos nas relações } \\
\text { com parceiros fixo }\end{array}$ & 89 & 56,3 & 69 & 43,7 & - & - & 158 & 100 \\
\hline $\begin{array}{l}\text { Tiveram relações com parceiros } \\
\text { casuais no último ano }\end{array}$ & 46 & 25,0 & 136 & 73,5 & 3 & 1,6 & 185 & 100 \\
\hline $\begin{array}{l}\text { Usaram preservativos nas relações } \\
\text { sexuais com parceiros casuais }\end{array}$ & 41 & 89,1 & 5 & 11,0 & - & - & 46 & 100 \\
\hline $\begin{array}{l}\text { Tiveram mais que um parceiro } \\
\text { sexual na vida }\end{array}$ & 118 & 64,0 & 66 & 35,7 & 1 & 0,5 & 185 & 100 \\
\hline
\end{tabular}

Nota:* Não Informou 
Dos 185 participantes com vida sexualmente ativa, $155(83,8 \%)$ eram do sexo feminino e 30 (16,2\%) do masculino. A Tabela 2 apresenta algumas práticas realizadas pelas universitárias (217) para o cuidado com a saúde sexual e prevenção de agravos à saúde. Pode ser observado que 89 $(41,0 \%)$ jovens realizaram o exame ginecológico no ano de 2015, 125 (57,0\%) realizaram o exame Papanicolau e $89(41,0 \%)$ fizeram o teste para o HIV. Entre as 155 respondentes sexualmente ativas, $12(7,7 \%)$ informaram o uso do preservativo feminino nos intercursos sexuais, enquanto $144(91,0 \%)$ afirmaram não terem feito o uso desse meio de prevenção. Apenas, $2(1,3 \%)$ não responderam à questão.

Tabela 2: Práticas de cuidado com a saúde sexual das graduandas de enfermagem. Rio de Janeiro, 2015.

\begin{tabular}{lcc}
\hline Perguntas somente para mulheres & f & $\mathbf{\%}$ \\
\hline Fez o exame ginecológico? & & \\
Neste ano & 89 & 41,0 \\
Ano passado & 49 & 22,6 \\
Há 1 ano & 20 & 9,2 \\
Nos últimos 2 anos & 10 & 4,6 \\
Há mais de 5 anos & 2 & 0,9 \\
Nunca fez & 19 & 8,8 \\
Não sabe/ Não informou & 28 & 12,9 \\
Total & 217 & 100,0 \\
\hline Fez o exame Papanicolau? & & \\
Sim & 125 & 57,6 \\
Não & 42 & 19,4 \\
Não lembra & 13 & 6,0 \\
Não informou & 37 & 17,1 \\
Total & 217 & 100,0 \\
\hline Já fez o teste para HIV alguma vez na vida? & \multicolumn{2}{c}{} \\
Sim & 89 & 41,0 \\
Não & 100 & 46,1 \\
Não informou & 28 & 12,9 \\
Total & 217 & 100.0 \\
\hline
\end{tabular}

Dentre os participantes do sexo masculino (33), $11(33,0 \%)$ realizaram a cirurgia de postectomia (circuncisão), e 21 (63,6\%) fizeram o teste para HIV pelo menos uma vez na vida. 
Entre os jovens homens sexualmente ativos (30), apenas $1(3,3 \%)$ teve relação sexual com mulher utilizando o preservativo feminino. A Tabela 3 apresenta os resultados das práticas de cuidado com a saúde empregada pelos universitários.

Tabela 3: Práticas de cuidado com a saúde dos graduandos de enfermagem do sexo masculino. Rio de Janeiro, 2015.

\begin{tabular}{lcc}
\hline Perguntas somente para os homens & $\mathrm{f}$ & $\%$ \\
\hline Já operou fimose ou fez circuncisão? & & \\
Sim & 11 & 33 \\
Não & 22 & 66,7 \\
Total & 33 & 100.0 \\
Já fez o teste para HIV alguma vez na vida? & & \\
Sim & 21 & 63,6 \\
Não & 12 & 36,4 \\
Total & 33 & 100.0 \\
${ }^{*}$ Já teve relação sexual com mulher usando preservativo feminino? & & \\
Sim & 1 & 3,3 \\
Não & 29 & 96,7 \\
Total & 30 & 100,0 \\
\hline * Os respondentes dessa pergunta foram somente os participantes sexualmente ativos & &
\end{tabular}

Nota: ${ }^{*}$ Os respondentes dessa pergunta foram somente os participantes sexualmente ativos

\section{Discussão}

O grupo investigado é composto, majoritariamente, por jovens do sexo feminino com idades entre 18 e 21 anos. Os achados estão em consonância com o Censo da Educação Superior de 2014 que sinaliza a predominância de mulheres na educação superior e a idade modal dos estudantes de 21 anos nos cursos presenciais. ${ }^{13}$ Considerando que a pesquisa foi realizada com estudantes de enfermagem, autores ressaltam que existe uma predominância feminina no processo de cuidar com a construção da profissão. ${ }^{14}$

Pesquisa avaliou como as mudanças no trabalho e na renda dos pais afetam as escolhas dos jovens, entre estudar e trabalhar, mostrou que quanto maior o rendimento dos pais, menor é 
a necessidade de manter os filhos trabalhando durante o processo de formação. ${ }^{15}$ Nos achados desta investigação evidenciou-se que maioria dos estudantes residia com seus pais e não trabalhava, especialmente, os participantes da universidade pública. Acrescenta-se que a graduação nessas universidades, em geral, ocorre em período integral, e os graduandos não conseguem conciliar o trabalho e, ao mesmo tempo, se dedicar ao curso. Esse fato faz com que haja necessidade do aporte financeiro dos familiares durante o seu processo de formação.

As atividades sexuais são uma prática inerente ao ser humano e requerem maturidade, responsabilidade e cuidados com a saúde para diminuir a vulnerabilidade às infecções transmitidas pelo sexo. O início das práticas sexuais de forma precoce e sem as orientações adequadas, aumenta a exposição dos jovens para contrair IST, em função da falta de maturidade cognitiva e emocional dessa população. ${ }^{8}$

Nesta pesquisa a faixa etária do início das atividades sexuais dos participantes foi entre 12 e 18 anos, o que está em consonância com estudos que sinalizam a idade média da iniciação sexual dos brasileiros aos 15 anos. ${ }^{16}$ Acrescenta-se que estão incluídos nessa faixa etária os estudantes do primeiro ano da graduação. $\mathrm{Na}$ ocasião, costumam vivenciar diversas transformações, como os relacionamentos afetivos e sexuais, que podem fomentar maior alternância de parceiros sexuais e favorecer a vulnerabilidade dos jovens às IST. ${ }^{17}$

O uso contínuo do preservativo é o principal método recomendado pelo Ministério da Saúde para a prevenção das IST. Nesta pesquisa constatou-se que, apesar de a maioria dos participantes utilizarem esse recurso na primeira relação sexual, não costumam adotar em todos os intercursos sexuais, o que contribui para que fiquem expostos às infecções transmitidas pelo contato sexual. Investigação ${ }^{18}$ realizada com adolescentes na faixa etária de 13 a 19 anos de uma escola pública, em Peruíbe-SP, com objetivo de comparar a vulnerabilidade ao HIV entre homens e mulheres, demonstrou que a frequência no uso do preservativo é maior entre os estudantes do sexo masculino. Já a relação do uso do preservativo em todos os intercursos sexuais, as estudantes do sexo feminino costumam adotar com maior frequência..$^{9,18}$ 
A maioria dos investigados informou ter se relacionado com parceiros fixos e fazer uso do preservativo. No entanto, um quantitativo expressivo de participantes mantém relacionamentos casuais, e não adotam o preservativo de modo continuo. Os achados evidenciam o uso de preservativos com parcerias fixas, no entanto, outras pesquisas mostram que o preservativo costuma ser negligenciado nesse tipo de relacionamento. Estudos ${ }^{9,18-19}$ sinalizam que os jovens envolvidos em relacionamentos com maior duração (parcerias fixas) tendem a desvalorizar o uso do preservativo, pois atribuem o seu uso a diminuição do prazer durante as relações sexuais ou associam seu uso a infidelidade do parceiro. O medo de prejudicar a relação é o principal motivo que leva os indivíduos em relacionamentos monogâmicos a negligenciar o uso do preservativo.

Nos achados observou-se que o preservativo masculino foi mais empregado pelos universitários que o preservativo feminino, embora o grupo investigado seja, majoritariamente, composto por mulheres. Estudo ${ }^{19}$ que analisou os motivos para não adesão ao preservativo feminino revelou que as mulheres costumam apresentar dificuldades na utilização e manuseio desse recurso, além de ter custo elevado quando comparado ao preservativo masculino. Ainda que o preservativo masculino seja o recurso mais utilizado, mesmo no grupo feminino, pesquisas ${ }^{18-19}$ indicam que o medo e a insegurança dificultam a negociação das mulheres com o parceiro para utilização do preservativo.

A realização do teste para detectar o HIV é garantida pela Portaria 29, de 17 de dezembro de $2013 .{ }^{20}$ Embora a população masculina apresente maior prevalência na realização do teste, estudos revelam que o acesso para as mulheres é maior quando comparado aos homens e que tem aumentado, principalmente, entre aquelas com melhor nível socioeconômico. ${ }^{21-22}$ Acrescenta-se que as unidades básicas no Brasil oferecem testes rápidos para detecção do HIV, da sífilis adquirida e das hepatites virais B e C, além de disponibilizar o tratamento para as pessoas com diagnóstico positivo. 
No tocante à circuncisão masculina, pesquisa $^{23}$ identificou que este procedimento apresenta um efeito protetor de $40 \%$ a $60 \%$ na redução do risco de exposição ao HIV nos homens heterossexuais, em áreas com alta prevalência do HIV (como a África). Demonstrou, também, os benefícios da circuncisão para a prevenção de infecções do trato urinário, de contrair o HIV, da transmissão de algumas IST (como o HPV - Human Papiloma Virus e HSV-2-Herpes simplex vírus type 2) e do câncer de pênis.

Em relação à realização do exame ginecológico, 63\% de mulheres investigadas realizou o exame recentemente. Estudos, ${ }^{22,24}$ contudo, sinalizam que a busca pelas mulheres para a realização de exames ginecológicos tem reduzido, em decorrência da falta de percepção de risco, de condições socioeconômicas, entre outros. É importante salientar que a realização do exame de Papanicolau envolve diversos fatores que favorecem ou não a adesão das mulheres, podendo-se elencar os trabalhos educativos e acolhimento dos profissionais de saúde, em especial dos enfermeiros, que favorecem a realização do exame, a presença de problemas vaginais e/ou recomendação médica. A vergonha, ansiedade, medo e insegurança, no entanto, são sentimentos que atuam como dificultadores e favorecem a não adesão das mulheres ao exame. Os profissionais de saúde, nesse contexto, têm um papel fundamental atuando como mediadores das necessidades desse contingente populacional, oferecendo suporte assistencial, orientações e esclarecimentos. Assim, de modo individual ou em grupo, com a adoção de estratégias educativas e respeitando as crenças e a cultura de cada mulher, esses trabalhadores podem contribuir de modo expressivo para a adesão ao exame, conscientizando-as sobre a importância da realização do exame preventivo, além de incentivar a procura pelas unidades de saúde. ${ }^{22,24}$

Os resultados obtidos nessa investigação são relevantes e demonstram a necessidade de discutir com a população jovem a importância da prática de sexo seguro para a prevenção de agravos para a sua saúde, como a ocorrência de uma IST. As instituições de ensino têm um papel fundamental, nesse contexto, contribuindo para a orientação dos estudantes sobre diversos assuntos, entre eles, a sexualidade. 
Cabe, ainda, acrescentar que os estudantes da área da saúde, em especial os de graduação em enfermagem, tem oportunidade de atuar durante a sua formação, em vários contextos como, também, na atenção primária de assistência a saúde. Nesse cenário tem-se o Programa Saúde nas Escolas (PSE) realizado em parceria do Ministério da Saúde e Ministério da Educação que, entre outros objetivos, busca reforçar a promoção da saúde e prevenção de doenças entre os estudantes da educação básica. O programa, em uma de suas dimensões, trabalha com a educação permanente e capacitação de profissionais e jovens, sob responsabilidade da Universidade Aberta do Brasil e do Ministério da Educação. Essa atividade tem interface com os Núcleos de Telessaúde do Ministério da Saúde e observa os temas e a constituição das equipes que irão trabalhar nos territórios dos Programas de Saúde da Família. Cabe ressaltar que, nessa dimensão, é previsto o monitoramento e a avaliação da saúde dos estudantes para sondagem de questões ligadas diretamente ao tema IST/Aids. ${ }^{25}$

Com essa iniciativa, nota-se a intenção do Governo Federal em oferecer aos estudantes orientação sobre a Educação em Saúde no contexto escolar. Esse programa, contudo, beneficia somente os alunos das escolas de ensino fundamental e médio da rede pública de ensino e adstritas à Estratégia da Saúde da Família. Os jovens de outras intuições de ensino e das universidades não são contemplados com as ações do PSE, contudo podem participar em ações do PSE na qualidade de acadêmicos bolsistas. Na perspectiva de prevenção de IST no grupo jovem, é importante que os serviços de atenção à saúde estejam articulados com as escolas e universidades, sendo espaços que favoreçam a interlocução e interação com os estudantes.

Considerando que o enfermeiro desempenha um importante papel no estímulo para a preservação da saúde sexual e reprodutiva dos jovens, engajado em atividades de educação em saúde, autocuidado, e suas ações contribuem para a redução da vulnerabilidade desse grupo às IST $^{10}$, seria oportuno que os graduandos, na qualidade de futuros profissionais de enfermagem, 
fossem estimulados para a assunção de práticas sexuais seguras considerando a preservação da saúde sexual.

Acrescenta-se que, no contexto da vulnerabilidade individual, os participantes apresentaram fragilidades em suas práticas sexuais e de cuidado com a saúde sexual, à medida que não adotam o preservativo de modo continuo em todos os intercursos sexuais, mesmo com parcerias casuais. Um quantitativo expressivo de jovens (42\%) não realizou o Papanicolau recentemente. É possível, portanto, que existam fatores não explícitos que interferem nas práticas sexuais e de cuidado para com a saúde dos jovens.

\section{Conclusão}

A pesquisa contou com a participação de estudantes do curso de graduação em enfermagem. Considerando serem universitários da área da saúde e o acesso a informação sobre a prevenção de infecções sexualmente transmissíveis, esperava-se que houvesse maior adesão e uso continuado do preservativo e de cuidados relativos à saúde sexual e reprodutiva. Os jovens adotam o preservativo masculino com maior frequência com parceiros casuais, em comparação com o uso com parceiros fixos, e uma parcela expressiva não adota em todos os intercursos sexuais.

Os resultados dessa investigação demonstram que os graduandos de enfermagem adotam comportamentos de risco em suas práticas sexuais, e se assemelham ao padrão de comportamento sexual de outros jovens na mesma faixa etária.

Práticas de educação em saúde realizadas nas universidades poderiam sensibilizar os estudantes sobre a importância da prática do sexo seguro, com estimulo ao uso do preservativo de modo continuo, mesmo com parceiros fixos, e dos cuidados com a saúde sexual. Essas ações, também, poderiam contribuir para a redução da vulnerabilidade dos universitários às IST e a assunção de um comportamento sexual com foco na preservação da saúde sexual. 
O estudo apresenta como limitação ter sido realizado apenas com estudantes de graduação em enfermagem. Seria oportuno que fosse replicado com estudantes de outras áreas de conhecimento e que os achados pudessem ser comparados.

\section{Referências}

1. Araújo MAL, Rocha AFB, Cavalcante EGF, Moura HJ, Galvão MTG, Lopes ACMU. Doenças sexualmente transmissíveis atendidas em unidade primária de saúde no Nordeste do Brasil. Cad Saúde Coletiva [Internet]. 2015 [acesso em 2018 fev 05];23(4):347-53. Disponível em: http://www.scielo.br/pdf/cadsc/v23n4/1414-462X-cadsc-23-4-347.pdf.

doi: http://dx.doi.org/10.1590/1414-462X201500040051.

2. Spindola T, Pimentel MRRA, Barros AS, Franco VQ, Ferreira LEM. Produção de conhecimento acerca das doenças sexualmente transmissíveis na população jovem: pesquisa bibliométrica. Rev Pesqui Cuid Fundamen Online [Internet]. 2015 [acesso em 2018 fev 05];7(3):3037-49. Disponível em: http://www.redalyc.org/pdf/5057/505750947029.pdf. doi: http://dx.doi.org/10.9789/21755361.2015.v7i3.3037-3049.

3. Tronco CB, Dell'Aglio DD. Caracterização do comportamento sexual de adolescentes: iniciação sexual e gênero. Gerais (Univ Fed Juiz Fora) [Internet]. 2012 [acesso em 2018 jun 15];5(2):254-69. Disponível em: http://pepsic.bvsalud.org/pdf/gerais/v5n2/v5n2a06.pdf.

4. Brasil. Lei n. 12.852, de 5 de agosto de 2013. Institui o estatuto da juventude e dispõe sobre os direitos dos jovens, os princípios e diretrizes das políticas públicas de juventude e o Sistema Nacional de Juventude - SINAJUVE [Internet]. 2013 ago 05 [acesso em 2018 jun 15]. Diário Oficial da União, Brasília (DF); 2013 ago 05. Seção 1, p. 1-4. Disponível em: http://www.in.gov.br/web/dou/-/lei-n-12-852de-5-de-agosto-de-2013-30042815.

5. Dantas KTB, Spindola T, Teixeira SVB, Lemos ACM, Ferreira LEM. Jovens universitários e o conhecimento acerca das doenças sexualmente transmissíveis: contribuição para cuidar em enfermagem. Rev Pesqui Cuid Fundamen Online [Internet]. 2015 [acesso em 2018 fev 05];7(3):3020-36. Disponível em: http://www.redalyc.org/html/5057/505750947028/. doi: http://dx.doi.org/10.9789/21755361.2015.v7i3.3020-3036.

6. Brasil. Ministério da Saúde. Secretaria de Vigilância em Saúde. Boletim Epidemiológico Sífilis 2017. Brasília (DF); 2017. [acesso em 2018 fev 05]. Disponível em: http://portalarquivos.saude.gov.br/images/pdf/2017/novembro/13/BE-2017-038-Boletim-Sifilis-11-2017publicacao-.pdf .

7. Brasil. Ministério da Saúde. Estudo epidemiológico sobre a prevalência nacional de infecção pelo HPV (POP-Brasil): resultados preliminares. Porto Alegre: Associação Hospitalar Moinhos de Vento; 2017. 
8. Costa ACPJ, Lins AG, Araújo MFM, Araújo TM, Gubert FA, Vieira NFC. Vulnerabilidade de adolescentes escolares às DST/HIV em Imperatriz - Maranhão. Rev Gaúcha Enferm [Internet]. 2013 [acesso em 2018 fev 05];34(3):179-86. Disponível em: http://www.scielo.br/pdf/rgenf/v34n3/a23v34n3.pdf.

9. D’Amaral HB, Rosa LA, Wilken RO, Spindola T, Pimentel MRAR, Ferreira LEM. As práticas sexuais dos graduandos de enfermagem e a prevenção das doenças sexualmente transmissíveis. Rev Enferm UERJ [Internet]. 2015 [acesso em 2018 fev 05];23(4):494-500. Disponível em: http://www.epublicacoes.uerj.br/index.php/enfermagemuerj/article/view/16823. doi: http://dx.doi.org/10.12957/reuerj.2015.16823.

10. Malagón-Oviedo RAM, Czeresnia D. O conceito de vulnerabilidade e seu caráter biossocial. Interface (Botucatu, Online) [Internet]. 2015 [acesso em 2018 fev 05];19(53):237-49. Disponível em: http://www.scielo.br/pdf/icse/2015nahead/1807-5762-icse-1807-576220140436.pdf. doi: http://dx.doi.org/10.1590/1807-57622014.0436.

11. Fonseca FF, Sena RKR, Santos RLAS, Dias OV, Melo S. As vulnerabilidades na infância e adolescência e as políticas públicas brasileiras de intervenção. Rev Paul Pediatr [Internet]. 2013 [acesso em 2018 fev 05];31(2):258-64. Disponível em: http://www.scielo.br/scielo.php?script=sci_arttext\&pid=S010305822013000200019. doi: http://dx.doi.org/10.1590/S0103-05822013000200019.

12. Ayres JRCM, França I, Junqueira G, Saletti HC. O conceito de vulnerabilidade e as práticas em Saúde: novas perspectivas e desafios. In: Czeresnia D, Machado C, organizadores. Promoção da Saúde: conceitos, reflexões, tendências. $2^{\mathrm{a}}$ ed. Rio de Janeiro: Fiocruz; 2009. p. 121-43.

13. Brasil. Ministério da Educação. Instituto Nacional de Educação e Pesquisa Educacionais Anísio Teixeira. Censo da Educação Superior. Brasília (DF): Ministério da Educação; 2015.

14. Souza LL, Araújo DB, Silva DS, Bêrredo VCM. Representações de gênero na prática de enfermagem na perspectiva de estudantes. Ciênc Cogn [Internet]. 2014 [acesso em 2018 fev 05];19(2):218-32. Disponível em: http://www.cienciasecognicao.org/revista/index.php/cec/article/view/908.

15. Vieira CS, Menezes-Filho N, Komatsu BK. Como as mudanças no trabalho e renda dos pais afetam as escolhas entre estudo e trabalho dos jovens. Pesquisa e Planejamento Econômico [Internet]. 2016 [acesso em 2018 fev 05];18(3):1-34. Disponível em: https://www.anpec.org.br/encontro/2015/submissao/files_I/i13-

a79a7f6f3956381a8015360db7d08141.pdf.

16. Pereira ALF, Penna LHG, Pires EC, Amado DC. Sexual and birth control health practices among female undergraduates: a descriptive study. Online Braz J Nurs [Internet]. 2014 [cited 2018 fev 05];4(23):25-35. Available from: http://www.objnursing.uff.br/index.php/nursing/article/view/4066/html_80.

17. Anjos RHD, Silva JAS, Val LF, Rincon LA, Nichiata LYI. Diferenças entre adolescentes do sexo feminino e masculino na vulnerabilidade individual ao HIV. Rev Esc Enferm USP [Internet]. 2012 [acesso em 2018 fev 05];46(4):829-37. Disponível em: 
http://bibliobase.sermais.pt:8008/BiblioNET/Upload/PDF5/003438_Rev\%20Esc\%20Enfer\%20USP\%206. pdf.

18. Oliveira LFR, Nascimento EGC, Pessoa Júnior JM, Cavalcanti MAF, Miranda FAN, Alchiere JC. Adesão de adolescentes à camisinha masculina. Rev Pesqui Cuid Fundamen Online [Internet]. 2015 [acesso em 2018 fev 05];7(1):1765-73. Disponível em: http://www.seer.unirio.br/index.php/cuidadofundamental/article/view/3467/pdf_1318.________ doi: http://dx.doi.org/10.9789/2175-5361.2015.v7i1.1765-1773.

19. Costa JES, Silva CD, Gomes VLO, Fonseca AD, Ferreira DA. Preservativo feminino: dificuldades de adaptação e estratégias para facilitar o uso rotineiro. Rev Enferm UERJ [Internet]. 2014 [acesso em 2018 fev 05];22(2):163-8. Disponível em: http://www.facenf.uerj.br/v22n2/v22n2a03.pdf.

20. Brasil. Ministério da Saúde. Secretaria de Vigilância em Saúde. Portaria № 29, de 17 de dezembro de 2013. Aprova o Manual Técnico para o Diagnóstico da Infecção pelo HIV em Adultos e Crianças e dá outras providências [Internet]. 2013 [acesso em 2018 fev 05]. Disponível em: http://bvsms.saude.gov.br/bvs/saudelegis/svs/2013/prt0029_17_12_2013.html.

21. Cárdenas CMM. Aconselhamento no âmbito dos Centros de Testagem no estado do Rio de Janeiro: uma análise das práticas e saberes na prevenção das DST/Aids [tese]. Rio de Janeiro (RJ): Instituto Oswaldo Cruz; 2014. Doutorado em Ciências na área de Saúde Pública.

22. Santos LV, Inagaki ADM, Abud ACF, Oliveira JKA, Ribeiro CJN, Oliveira MIA. Características sociodemográficas e risco para doenças sexualmente transmissíveis entre mulheres atendidas na atenção básica. Rev Enferm UERJ [Internet]. 2014 [acesso em 2018 fev 05];22(1):111-5. Disponível em: https://www.e-publicacoes.uerj.br/index.php/enfermagemuerj/article/view/11456.

23. American Academy of Pediatrics. Male circumcision. Task force on circumcision. Pediatrics [Internet]. 2012 [cited 2018 Jun 15]. 130 Spec:756-85. Available from: http://pediatrics.aappublications.org/content/pediatrics/130/3/e756.full.pdf.

24. Oliveira AEC, Deininger LSC, Lima IMB, Lima DC, Nascimento JA, Andrade JM. Adesão das mulheres ao exame citológico do colo uterino na atenção básica. Rev Enferm UFPE On Line [Internet]. 2016 [acesso em 2018 fev 05];10(11):4003-14. Disponível em: http://pesquisa.bvsalud.org/enfermeria/resource/pt/bde-30146.

25. Brasil. Ministério da Saúde. Ministério da Educação. Passo a passo PSE: Programa Saúde na Escola: tecendo caminhos da intersetorialidade. Brasília (DF): Ministério da Educação; 2011. [acesso em 2018 fev 05]. Disponível em: http://bvsms.saude.gov.br/bvs/publicacoes/passo_a_passo_programa_saude_escola.pdf.

\section{Autor correspondente}

Thelma Spindola

E-mail: tspindola.uerj@gmail.com 
Endereço: Boulevard 28 de Setembro, 157 - Vila Isabel, Rio de Janeiro - RJ

CEP: 20551-030

\section{Contribuições de Autoria}

\section{1 - Thelma Spindola}

Concepção do projeto, análise e interpretação dos dados, redação do artigo e revisão crítica do conteúdo intelectual.

\section{2 - Carolina Passos Sodré}

Coleta dos dados, análise e interpretação dos dados, redação do artigo.

\section{3 - Cláudia Silvia Rocha Oliveira}

Análise e interpretação dos dados, redação do artigo e revisão crítica do conteúdo intelectual

4 - Barbara Ingênito de Oliveira

Coleta dos dados, análise e interpretação dos dados, redação do artigo.

\section{5 - Rosana Santos Costa Santana}

Redação do artigo e revisão crítica do conteúdo intelectual.

\section{6 - Nathalia Lourdes Nepomuceno de Oliveira André}

Coleta dos dados, interpretação dos dados e redação do artigo.

\section{Como citar este artigo}

Spindola T, Sodré CP. Oliveira CSR, Oliveira BI, Santana RSC, André NLNO. Práticas sexuais e cuidados relacionados à saúde sexual de graduandos de enfermagem frente às infecções sexualmente transmissíveis. Rev. Enferm. UFSM. 2019 [Acesso em: 2019 jun 15];vol ex:1-18. DOI:https://doi.org/10.5902/2179769231117 